\title{
Path-Sensitive Region Analysis for Mercury Programs
}

\author{
Quan Phan Gerda Janssens \\ Department of Computer Science, K.U.Leuven \\ Celestijnenlaan, 200A, B-3001 Leuven, Belgium \\ \{quan.phan,gerda.janssens\}@cs.kuleuven.be
}

\begin{abstract}
Dividing the heap memory of programs into regions is the starting point of region-based memory management. In our existing work of enabling region-based memory management for Mercury, a program analysis was used to distribute data over the regions. An important goal of the analysis is to decide which program variables should end up in the same region. For a popular class of programs, it covetously puts program variables in the same region, while more memory could have been reused if they had been kept in separate ones. In this paper we define a new refined region analysis that is keen to keep program variables in separate regions by taking into account the different execution paths of a procedure. With the more precise, path-sensitive analysis we can reduce the memory footprint for several programs.
\end{abstract}

Categories and Subject Descriptors $\quad$ D.3.4 [Processors]: Compilers, Memory management (garbage collection)

General Terms Languages, Performance

Keywords Region analysis, region-based memory management, region reuse, logic programming, Mercury

\section{Introduction}

Logic programming languages have a long tradition of freeing programmers from procedural chores such as manual memory management. A recent approach to automated memory management apart from runtime reference-tracing garbage collection is to rely on static analysis and program transformation that can approximate lifetime of program data and instruct the program to reuse dead memory at runtime. Region-based memory management (RBMM) follows this approach. It is based on statically dividing the heap memory into different parts, called regions, in which program terms are stored. Then the aim is to arrange this in such a way that the memory occupied by dead terms can simply be released by reclaiming their region as a whole. Recently region-based memory management has been made available in several mainstream logic programming systems, such as the WAM-based Prolog system reported in [3] and the Melbourne Mercury Compiler in [4, 6].

In [4] and [6] we have developed an RBMM system for Mercury. The static region analysis and transformation were given in [4], which annotate programs with region instructions that take

Permission to make digital or hard copies of all or part of this work for personal or classroom use is granted without fee provided that copies are not made or distributed for profit or commercial advantage and that copies bear this notice and the full citation on the first page. To copy otherwise, to republish, to post on servers or to redistribute to lists, requires prior specific permission and/or a fee.

PPDP'09 September 7-9, 2009, Coimbra, Portugal.

Copyright (C) 2009 ACM 978-1-60558-568-0/09/09 . .\$10.00 care of the timely creation and removal of regions and also of the adequate distribution of the terms over the regions. In [6] we described the runtime support needed for RBMM that could also handle backward execution. Although the system gave very promising results, in terms of memory consumption as well as runtime speedup, for almost all of the benchmarks [6], the static region analysis in [4] sometimes too eagerly grouped variables into regions without taking into account different execution paths, reducing reuse opportunities. Our contribution in this paper is the improvement of the region analysis and transformation in [4]. By distinguishing execution paths we obtain a more precise region allocation that ultimately leads to better memory reuse.

Section 2 motivates our new approach of dealing with different execution paths. Section 3 describes how we use, just as in [5], type-based graphs to model the regions of types. The concept of region points-to graphs and its extension with same-edges to keep apart regions are in Section 4. The region points-to analysis, Section 5, computes a safe region model and now is linked up with region liveness analysis in Section 6 to ensure the safety of sameedges. This link is described in Section 7. In Section 8 we show the impact of same-edges on the program transformation. Section 9 discusses and concludes.

\section{Motivation}

We assume the reader is familiar with Mercury [7]. The explicit declarations of types and modes in Mercury enable its compiler to convert all predicate definitions into procedures in superhomogeneous form in which unifications are specialized into $<=$ for construction unifications, $\Rightarrow$ for deconstruction unifications, $==$ for equality tests, and := for assignments.

We will look at the relation between assignments and regions in particular. Consider the assignment $X:=Y$, which binds the free variable $X$ to the value of $Y$. For now we assume that the value of the variable $\mathrm{Y}$ is simply stored in one region. (We come back to the issue of storage of variables later in Section 3). As after the assignment $\mathrm{X}$ and $\mathrm{Y}$ are bound to the same value in a region, we can say that the two variables are in the same region.

The code in Figure 1 is a part of a Mercury program that manipulates lists of integers. We associate a program point with every literal (i.e., a specialized unification or a procedure call) in the body of a procedure. An execution path is a sequence of program points, such that at runtime the literals associated with these program points are performed in sequence. The procedure $\mathrm{q}$ has two execution paths: $\langle(1),(2)\rangle$ and $\langle(3),(4)\rangle$ in which different assignments for $\mathrm{Z}$ occur. Note that the program point (1) does not belong to the second execution path because for an if-then-else if $C$ then $T$ else $E$ Mercury will first try $C$ but if $C$ fails, it will ensure that $E$ is executed as if $C$ had never been tried. In the second execution path $q$ invokes produce that can assumably put its output argument $\mathrm{Y}$ in a region different from that of $\mathrm{X}$. 


\begin{tabular}{|ll|}
\hline$\%$ (in, in, out). & $\%$ (in, out) \\
$\mathrm{q}(\mathrm{N}, \mathrm{X}, \mathrm{Z}):-$ & produce $(\mathrm{X}, \mathrm{Y}):-$ \\
( if & $($ (1) $\mathrm{X}=\mathrm{C}$, \\
(1) $\mathrm{N}>0$ & $(2) \mathrm{Y}<=[]$ \\
then & $;(3) \mathrm{X}=\mathrm{XX} \mid \mathrm{Xs}]$ \\
(2) $\mathrm{Z}:=\mathrm{X}$ & (4) produce(Xs, Ys) \\
else & (5) $\mathrm{Y}<=[\mathrm{Xe}+1 \mid \mathrm{Ys}]$ \\
(3) produce $(\mathrm{X}, \mathrm{Y})$, & ). \\
(4) $\mathrm{Z}:=\mathrm{Y}$ & \\
\hline
\end{tabular}

Figure 1: The running example.

The code of produce in Figure 1 follows this pattern. If after a call to produce its input is no longer needed, we can reclaim its memory by removing its corresponding region, without affecting the output. The call to produce at (3) in q is such a call. In q, after the assignment at (2) in the first execution path, that $\mathrm{Z}$ is bound to the list bound to by $\mathrm{X}$ implies that these two variables are in the same region. Similarly, at (4) in the second execution path we have that $\mathrm{Z}$ and $\mathrm{Y}$ are in the same region. So it seems reasonable and is actually safe to put all $\mathrm{X}, \mathrm{Y}$ and $\mathrm{Z}$ in one region.

The region points-to analysis in [4] follows this "eager" approach. So if the program follows the second execution path of q, the eager approach prohibits the reclamation of the memory of $\mathrm{X}$, even when it is dead after the call to produce (recall that we assume that $\mathrm{X}$ and $\mathrm{Y}$ are independent). The memory for $\mathrm{X}$ could have been reclaimed if it had been kept apart in a different region from that of $Y$ and $\mathrm{Z}$.

Let us explore the idea of keeping apart the regions for $\mathrm{X}, \mathrm{Y}$ and $\mathrm{Z}$ in order to be able to reclaim memory better. Thus we would like to keep $\mathrm{X}, \mathrm{Y}$ and $\mathrm{Z}$ in different regions. An assignment like $\mathrm{Z}:=$ $X$ could then involve the copying of the value of $X$ into the region of $\mathrm{Z}$. Although this allows us to reclaim the memory of $\mathrm{X}$ in the else branch it incurs the overhead due to the copying, which is not desirable as it is linear to the size of the value. We can do better by reusing the region of $\mathrm{X}$ as the region of $\mathrm{Z}$ at (2) and the region of $\mathrm{Y}$ as the region of $\mathrm{Z}$ at (4), while the memory of $\mathrm{X}$ is reclaimed in produce as shown in the region-annotated code in Figure 2. We can view this as the result of distinguishing execution paths

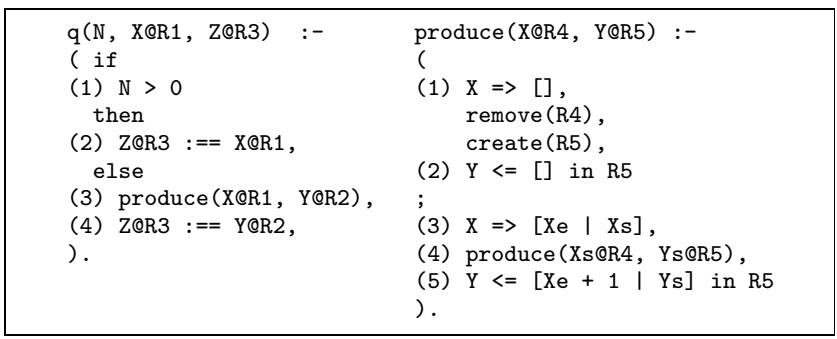

Figure 2: Region-annotated version.

of a procedure when dividing its variables into regions. In the first execution path, $\mathrm{X}$ and $\mathrm{Z}$ are in the same region while in the second one, $\mathrm{X}$ is in a region different from that of $\mathrm{Y}$ and $\mathrm{Z}$.

In the region-annotated version, we use region variables R's to refer to regions. The region instruction create $(R)$ creates a region and makes $R$ bound to it while remove $(R)$ removes the region that $R$ is bound to. The special region-annotated assignment notation $\mathrm{X} @ \mathrm{R} 1:==\mathrm{Y} @ \mathrm{R} 2$ means that $\mathrm{R} 1$ is bound to the region of $\mathrm{R} 2$, then $Y$ is assigned to $X$, after that R2 is unbound. Its effect is that we reuse the region of $\mathrm{R} 2$ for $\mathrm{R} 1$. We use $\mathrm{QR}$ to illustrate the passing of regions as arguments and annotate construction unifications with in $\mathrm{R}$, the region into which the allocation happens. Then the behaviour of the code is as follows. A caller of q prepares $X$ in the region bound to by $\mathrm{R} 1$ and expects $\mathrm{Z}$ in some region bound to by R3. If the first execution path of $q$ is taken $R 3$ will be bound to the region $\mathrm{R} 1$. If the second path is, the call to produce removes $\mathrm{R} 1$ and creates $\mathrm{R} 2$ in its base case, then $\mathrm{R} 3$ is bound to R2.

\section{Modelling the Regions Based on Types}

In this section we explain the relationship between types and the storage of program variables in regions.

We use the type of a variable, which determines its values, to distribute such a value (i.e., the term bound to by the variable) over several regions. A list is stored in two regions: one for the listskeleton and one for the elements of the list. The benefit of such a distribution is that a program, such as quicksort and naive reverse on lists, often creates several temporary lists but the elements of the input list are needed throughout. When a temporary list is no longer needed, its list-skeleton as a whole can be freed by one single action namely by removing its region.

The scheme of regions used to store terms of a type can be derived systematically from the type declaration [5]. Consider the following type declarations in which int is a builtin type in Mercury.

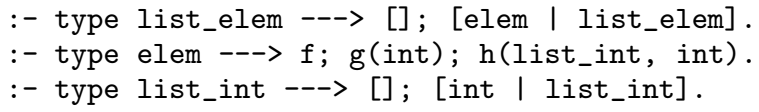

The type list_elem has two principal functors, []$/ 0$ and $[\mathrm{I}] / 2$. We store these principal functors in a region $r 1$. This means that the memory block needed to represent e.g., [I] $/ 2$ is allocated into $r 1$. The block contains tagged pointers to the arguments of $[\mathrm{I}] / 2$. The first argument is of type elem different from list_elem, therefore it is stored into a different region $r 2$. The second argument is again of type list_elem and we also store it in the region $r 1$. In this way the list skeleton as a whole is in one region. The type elem has three principal functors: $f / 0, g / 1$ and $h / 2$. Suppose $L$ has type list_elem and is bound to $[f, g(1), h([1,2], 2)]$. To represent this list $\mathrm{L}$, we need three regions shown by the dash boxes in Figure 3. In the first we store the principal functors of list_elem,

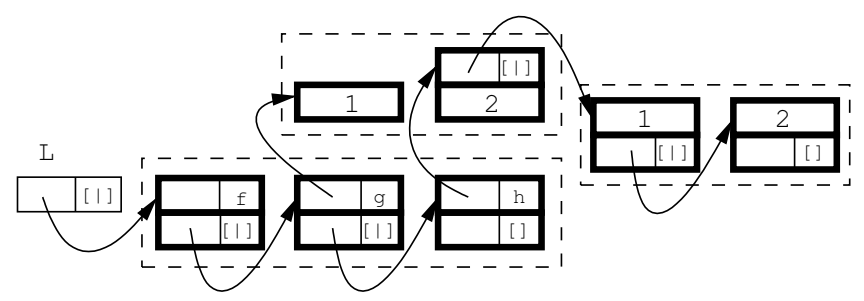

Figure 3: Region storage of the term bound to by L.

the so-called list skeleton. The elements of the list are stored in the second region, in particular the principal functors of the elem terms. Some arguments of the principal functors of elem have type int that does not require extra memory so they are in the memory blocks of the $g / 1$ and $h / 2$ functors. The first argument of $h / 2$ is a list of int's and needs one more region.

For each type we define a memory-storage scheme modelled by a type-based region graph $T G(N, E)$ with $N$ a set of nodes and $E$ a set of directed edges. A node stands for a region variable. Just as program variables get bound to ordinary Mercury terms during the execution of a program, region variables will get bound to (physical) regions. A directed edge from one node to another represents the fact that the region bound to by the region variable represented by the former node contains references into (points-to) 
the region bound to by the region variable represented by the latter one. The reference relation represented by the edges is actually defined by the type.

The type-based region graph for the type list_elem is shown in Figure 4 . The [I] principal functor is stored in $R^{\text {list_elem }}$. Actually we need a block of two memory words. [I] has two arguments, the first having the type elem and the second having the same type list_elem. Thus we have two edges from $R^{\text {list_elem }}$, one pointing to $R^{\text {elem }}$ where the principal functors of elem $(\mathrm{g} / 1$ and $\mathrm{h} / 2)$ are stored and the other is a self-edge. The edge labelled $(h, 1)$ is due to the first argument of the functor $\mathrm{h} / 2$.

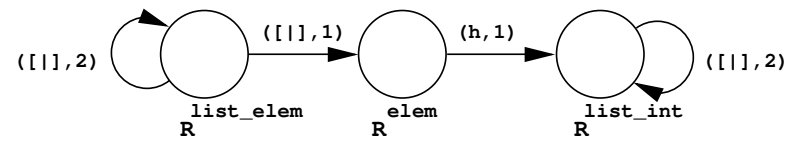

Figure 4: The type-based region graph of the type list_elem.

Consider the following type:

:- type $t-->\ldots ; f(t 1, \ldots, t i, \ldots, t n) ; \ldots$

If $R^{t}$ is represented by the node $n$, then we have for each type ti that needs heap storage a node $m$ representing $R^{t i}$ and exactly one edge $(n,(f, i), m)$ with the label $(f, i)$. We refer to $n$ as the principal node of $T G_{t}$.

Mercury has polymorphic types but we can safely just ignore type variables in our region model because procedures that work with polymorphic types will never actually manipulate the concrete terms corresponding to the type variables.

During the execution of the program, the regions used to allocate terms belonging to a type $t$, will be an instance of the memorystorage scheme modelled by $T G_{t}$, the type-based region graph for $t$.

\section{Region Points-to Graph}

We use the notion of a region points-to graph to model the memory used by a Mercury procedure. We use the set of type-based region graphs, one for each variable of the procedure, to adequately model the memory locations of the procedure's terms, However, we also need to model the sharing of (sub)terms among the program variables, which is created during the execution of the procedure. One of the contributions of this paper is the modelling of this sharing.

In Mercury, the instantiation of variables, therefore the sharing among them, is caused by unifications. We divide the sharing into two groups. First, a construction unification $X<=f(\ldots, Y$, ...) allocates new memory for storing the functor $f$ (actually the block of memory words corresponding to $f$ ) and creates sharing between $X$ and $Y$. Also in a deconstruction unification $X \Rightarrow f(\ldots$, $\mathrm{Y}, \ldots) \mathrm{Y}$ is instantiated and $\mathrm{Y}$ shares with a subterm of $\mathrm{X}$. The regions needed to store the values of $\mathrm{X}$ are given by its type-based region graph in which the edges point into the regions of subterms. In order to represent this sharing between $\mathrm{X}$ and $\mathrm{Y}$, we do as follows. A node $n$ is associated with a set of program variables, $\operatorname{vars}(n)$, whose principal functors are stored in the region that is bound to by the region variable that is represented by $n$. The sharing between X and Y (with Y the $i^{\text {th }}$ argument of X) is then represented by having a node $n_{X}$ with $X \in \operatorname{vars}\left(n_{X}\right)$, an edge $\left(n_{X},(f, i), n_{Y}\right)$ and $Y \in \operatorname{vars}\left(n_{Y}\right)$. The vars set of a node can contain either zero, one or more variables. In the case where constructions and deconstructions involve variables of recursive types such as lists, e.g., L $<=[\mathrm{E} \mid \mathrm{T}]$ or $\mathrm{L} \Rightarrow[\mathrm{E} \mid \mathrm{T}], \mathrm{L}$ and $\mathrm{T}$ are forced to end up in the same vars set. All the program variables in the vars set of a node may be allocated in the same region.
Second, an assignment unification $\mathrm{X}:=\mathrm{Y}$ binds $\mathrm{X}$ to $\mathrm{Y}$ and creates sharing between $X$ and Y. Previously in [4], we did put the two variables in the same region because they actually point to the same term after the assignment. In the new approach in this paper we keep their regions apart and just remember that they are candidates for reusing one for the other after this point. We represent this in a region points-to graph by a new kind of edges, called same-edges. The sharing created by X $:=\mathrm{Y}$ is modelled by a directed edge $s\left(n_{Y}, i, n_{X}\right)$ with $i$ the program point where $\mathrm{X}:=$ $\mathrm{Y}$ is found. Note that normally the same-edge between $n_{X}$ and $n_{Y}$ should propagate to the regions of their corresponding subterms.

A region points-to graph $G(N, E, S)$ for a set of variables $V$ consists of a set of nodes, $N$, representing region variables, a set of directed edges, $E$, representing references between the regions bound to by these region variables and a set of directed same-edges, $S$, to model candidates for reuse. The nodes are annotated with vars sets: we have $V=\bigcup_{n \in N} \operatorname{vars}(n)$. The vars sets are disjoint. The node $n_{X}$ denotes the node such that $X \in \operatorname{vars}\left(n_{X}\right)$. The function node $\left(n_{X},(f, i)\right)$ returns the node $m$ if $\left(n_{X},(f, i), m\right) \in$ $E$ otherwise it is undefined. The edges in $S$ model regions that are possible candidates for reuse. Whether the reuse can safely be done depends on the liveness of the involved regions. This will become clear later.

The region points-to graphs of the procedures $q$ and produce in our running example are in Figure 5. With same-edges we can keep

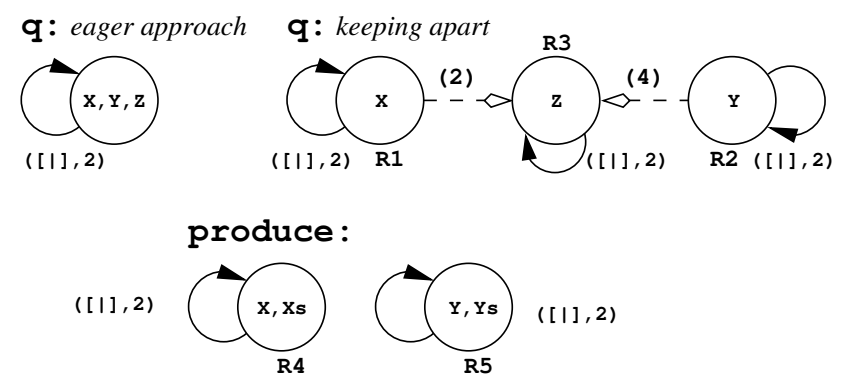

Figure 5: Region points-to graphs of $\mathrm{q}$ and produce.

the regions of $\mathrm{X}, \mathrm{Y}$, and $\mathrm{Z}$ separate, in contrast to the eager approach where we would have only one node with these variables in its vars set. Now we are still able to capture the fact that at some program points (i.e., at (2) and (4)) they are the same. Note again that we assume no regions for the integer elements of the list because they are stored right in the first word of a cons cell.

All in all, sharing is represented in a region points-to graph in three ways. Firstly, the directed edges in $E$ represent sharing of subterms. Secondly, that a vars set of a node may contain more than one variable represents the fact that these variables may be bound to the same term or to different subterms of a recursive term. Finally, sharing due to assignments is represented by the sameedges in $S$.

\section{Region Points-to Analysis}

The region points-to analysis computes a region model for a procedure and the whole program by capturing the locations and the sharing among variables. To capture sharing we use two operations: unify and same_edge. The operation unify is defined in Algorithm 1. Unifying two nodes $n$ and $m$ implies that the variables in $\operatorname{vars}(n)$ and those in $\operatorname{vars}(m)$ are stored in the same region. To ensure that there is only one out-edge with a specific label from one node to another the operation is recursive, i.e., unifying two nodes may cause more nodes to be unified. 


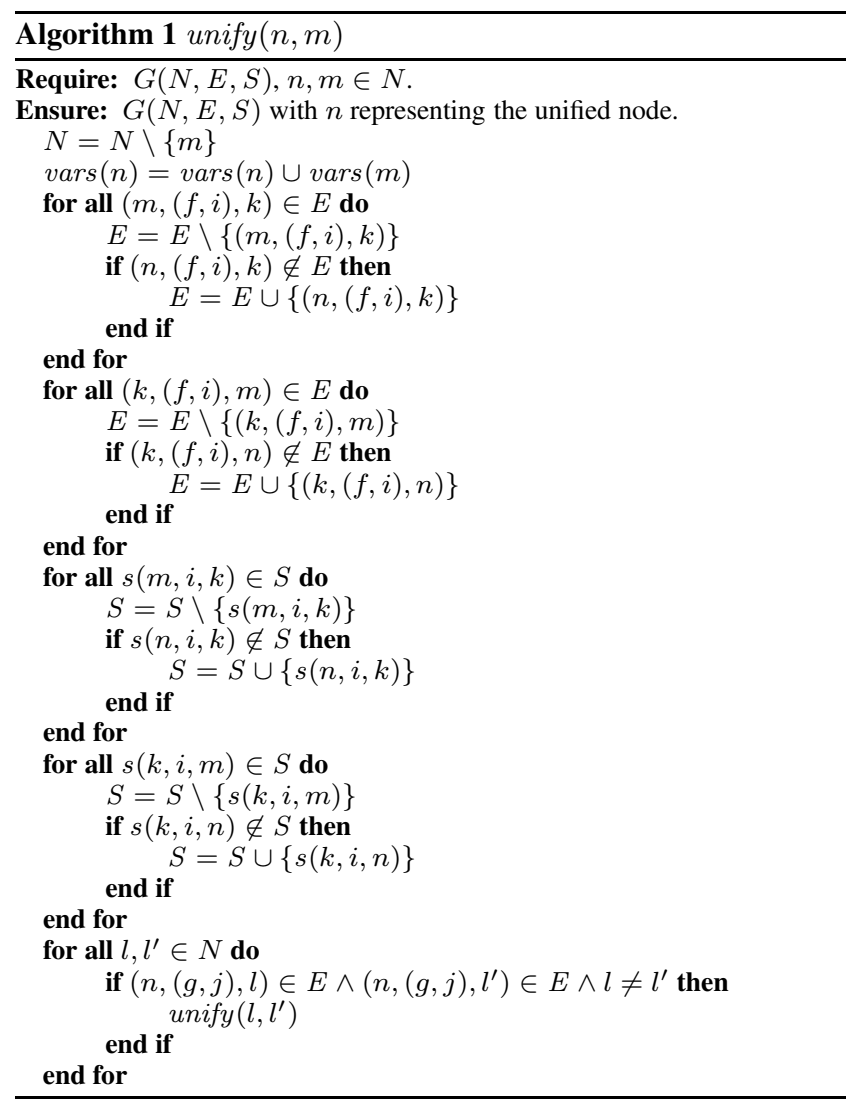

The novelty in this paper (compared to [4]) is the recording of sharing by using the same_edge operation that is defined by Algorithm 2. When we record a same-edge between two nodes we

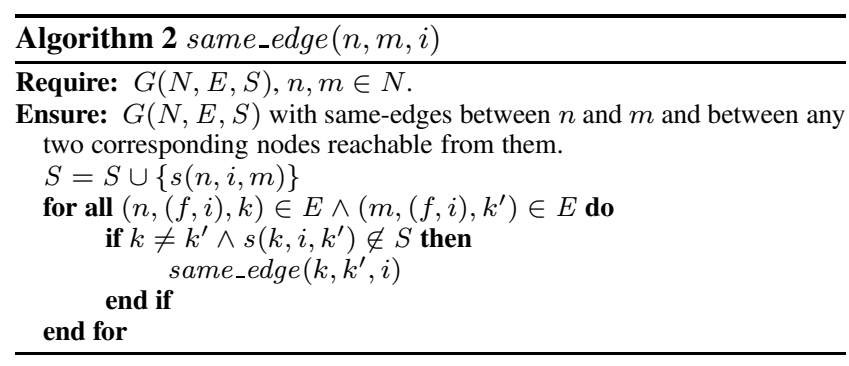

also need to recursively record same-edges for the corresponding nodes reached from them through corresponding edges. Recall that by $s\left(k, i, k^{\prime}\right)$ we mean to reuse the region bound to by the region variable represented by $k$ for the region variable represented by $k^{\prime}$ at the program point $i$.

\subsection{Intraprocedural Analysis of a Procedure}

The intraprocedural analysis initializes $G_{p}$ and then captures the sharing created by the explicit unifications. Its definition is in Algorithm 3 . The function $p p(l)$ returns the program point associated to the literal $l$.

As we know the type of each variable in $p$, we initialize $G_{p}$ by using the type-based region graphs $(T G)$ of the variables. In Algorithm 3, we use the function init_rptg $(X)$ that generates a region points-to graph for $X$ from the type-based region graph of the type of $X, T G_{\text {type }(X)}$, by copying all the nodes and edges, but initializing the vars set of the node corresponding to the principal

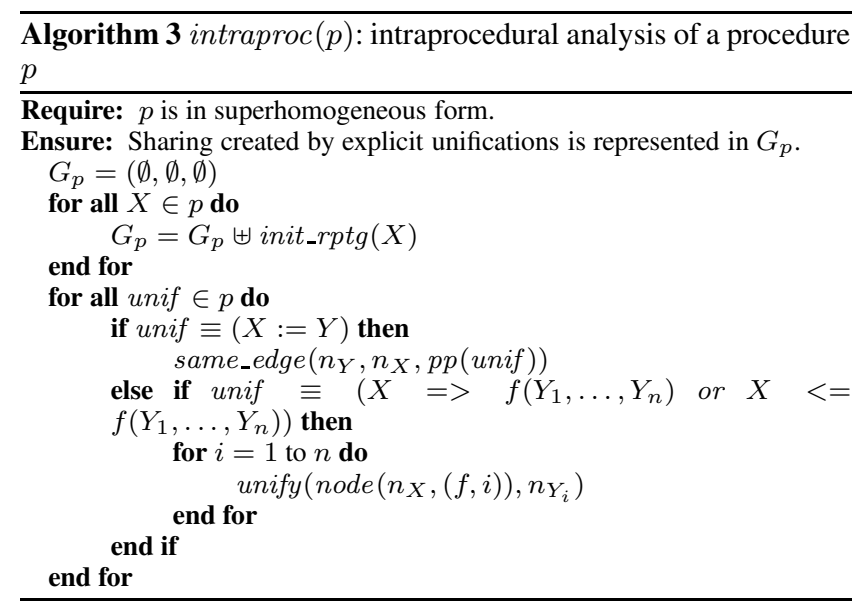

node in $T G_{\text {type }(X)}$ with $\{X\}$ and those of the other nodes with an empty set, generating a fresh region variable for each node in the region points-to graph, and setting the set of same-edges to empty. The region points-to graph returned by init_rptg $(L)$, with $\mathrm{L}$ of the type list_elem (Figures 3 and 4) is shown in Figure 6 with the assumption that the region variables are fresh.

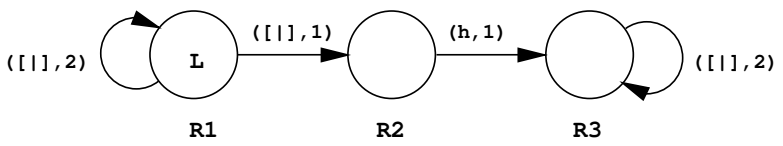

Figure 6: The resulting region points-to graph of init_rptg $(L)$.

The intraprocedural analysis adds all the sharing created by the unifications in the procedure to $G_{p}$. We ignore test unifications because they do not create any sharing. For construction and deconstruction unifications we unify the nodes corresponding with the sharing created by them. For an assignment we say that at its program point the two variables are bound to the same term by adding a same-edge from the node of the right-hand side variable to the node of the left-hand side one. This leaves the possibility that in other execution paths they are not necessarily bound to the same term, therefore they do not necessarily have to be in the same region either.

\subsection{Interprocedural Analysis}

The interprocedural analysis, Algorithm 4, updates $G_{p}$ by integrating the relevant sharing information from the region points-to graphs of the called procedures into $G_{p}$.

For a call $q\left(Y_{1}, \ldots, Y_{n}\right)$, the head of the defining procedure is assumed to be $q\left(X_{1}, \ldots, X_{n}\right)$. The sharing among $X_{i}$ 's in $G_{q}$ may not have been present in $G_{p}$ as sharing among $Y_{i}$ 's. The interprocedural analysis makes sure that this will be the case. Firstly, it builds the function $\alpha: N_{q} \rightarrow N_{p}$ that maps the nodes of the formal arguments $\left(X_{i}\right.$ 's) to the nodes of the corresponding actual arguments $\left(Y_{i}\right.$ 's). Then these nodes are the starting points for the integration of the remaining sharing. This is done by following the relevant edges in $G_{q}$ to extend the $\alpha$ function to all the relevant nodes in $G_{q}$ (rule $\mathrm{P} 2$ ) and to unify the relevant nodes in $G_{p}$ (rule P1). Then we export the same-edges by relying on the $\alpha$ function at the call site. The program point of the same-edges is the program point of the call.

For a whole program, we can first do the intraprocedural analysis for every procedure. Then given the fact that in the interprocedural analysis the analysis information is only propagated from graphs of callees to those of callers, we can do the interprocedural analysis for a program efficiently by decomposing the call-dependency 


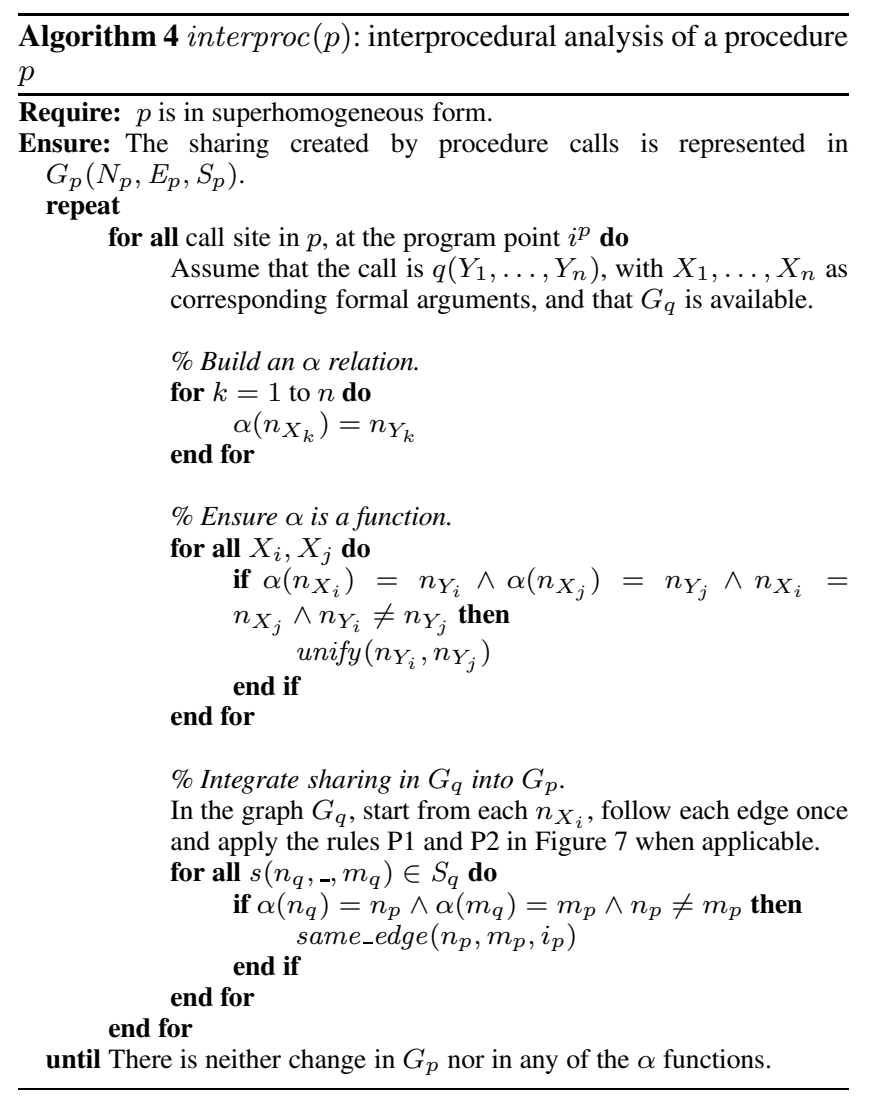

\begin{tabular}{|c|c|c|c|}
\hline \multirow{2}{*}{$\begin{array}{c}\left(n_{q},(f, i), m_{q}\right) \in E_{q} \\
\alpha\left(n_{q}\right)=n_{p} \\
\left(n_{p},(f, i), m_{p}^{\prime}\right) \in E_{p} \\
\alpha\left(m_{q}\right)=m_{p} \neq m_{p}^{\prime} \\
\text { unify }\left(m_{p}, m_{p}^{\prime}\right)\end{array}$} & \multirow{2}{*}{ (P1) } & $\begin{array}{l}\left(n_{q},(f, i), m_{q}\right) \in E_{q} \\
\alpha\left(n_{q}\right)=n_{p} \\
\left(n_{p},(f, i), m_{p}\right) \in E_{p} \\
\alpha\left(m_{q}\right) \text { undefined }\end{array}$ & \multirow{2}{*}{ (P2) } \\
\hline & & $\alpha\left(m_{q}\right)=m_{p}$ & \\
\hline
\end{tabular}

Figure 7: Interprocedural analysis rules.

graph into a tree of strongly connected components, and analysing the components in bottom-up order.

The result of the region points-to analysis for the running example in Figure 1 has been shown in Figure 5. In the graph for produce, that $\mathrm{X}$ and $\mathrm{Xs}$ are in $\operatorname{vars}\left(R_{4}\right)$ and that $\mathrm{Y}$ and $\mathrm{Ys}$ are in $\operatorname{vars}(R 5)$ are due to the deconstruction at (3) and the construction at (5), respectively. Analysing the recursive call at (4) records $\alpha\left(R_{4}\right)=R_{4}$ and $\alpha(R 5)=R 5$ but makes no change to the shape of the graph. In the graph for q, the two same-edges are due to the assignments at (2) and (4). Processing the call to produce at (3) creates $\alpha(R 4)=R 1$ and $\alpha(R 5)=R 2$.

\section{Region Liveness Analysis}

After the region points-to analysis we know the region variables of each procedure and how the program variables are distributed over the regions to which these region variables are bound. As regions may need to exist through a sequence of procedure calls, e.g., a call may allocate memory into an existing region, we do pass region variables as arguments of procedures. We use the existing region liveness analysis $[4,5]$ to decide which region variables are live at each program point and which region arguments become live or stop to be live in each procedure. In this section we summarize the relevant notions. Within the scope of a procedure we determine the local liveness of (program) variables and region variables in Section 6.1. The global liveness is discussed in Section 6.2.

\subsection{Live Variables and Live Region Variables}

We use the notions before and after a program point. Before a program point means right before the associated literal is going to be executed; while after a program point means its literal has just been completed. A program variable is live before a program point if it has been instantiated before the point and may be used in the future. A program variable is live after a program point if it has been instantiated before or at the point and may be used in the future. The live variable analysis computes for each program point $i$ the set of variables that are live before $i, L V_{\text {before }}(i)$, and the set of variables that are live after $i, L V_{\text {after }}(i)$. The $L V_{\text {before }}$ of the first program point(s) in a procedure $\mathrm{p}$ is defined to be the set of input arguments of $\mathrm{p}$, in_args $(p)$, while the $L V_{\text {after }}$ of the last program point(s) in a procedure $\mathrm{p}$ is defined to be the set of output arguments of $\mathrm{p}$, out_args $(p)$.

A region variable being live means that it should be bound to a region and that the region variable is possibly used in future (forward) execution. A region variable is live before (after) a program point if its node is reachable from a variable that is live before (after) the program point.

The set of nodes that are reachable from a variable is defined as follows.

$$
\operatorname{Reach}(X)=\left\{n_{X}\right\} \cup\left\{m \mid \exists\left(n_{X}, m\right) \in E^{*}(X)\right\},
$$

in which $E^{*}(X)$ is defined:

$$
E^{*}(X)=\left\{\left(n_{X}, n_{i}\right) \mid \exists\left(n_{X}, \text { label }_{0}, n_{1}\right), \ldots,\left(n_{i-1}, \text { label }_{i-1}, n_{i}\right) \in\right.
$$

The live region variables sets before and after a program point $i$ are defined:

$$
\begin{gathered}
L R_{\text {before }}(i)=\bigcup_{X \in L V_{\text {before }}(i)} \operatorname{Reach}(X) . \\
L R_{\text {after }}(i)=\bigcup_{X \in L V_{\text {after }}(i)} \operatorname{Reach}(X) .
\end{gathered}
$$

\subsection{Lifetime of Regions across Procedure Boundary}

Region arguments are used to pass regions among procedure calls in order to achieve better memory reuse by keeping the lifetime of regions short. Therefore, the global liveness analysis part derives which region variables become live or cease to be live inside a procedure.

Consider a procedure $q$ that is called by some procedure $p$, we define:

- $\operatorname{bornR}(q)$ is the set of region variables of $q$ that are mapped (by the $\alpha$ function at the call site) to region variables of $p$ that definitely become live inside $q$, i.e., in $q$ or in one of the procedures it calls.

- $\operatorname{deadR}(q)$ is the set of region variables of $q$ that are mapped to region variables of $p$ that definitely cease to be live (or become dead) inside $q$.

- outlivedR $(q)$ is the set of region variables of $q$ that are mapped to region variables of $p$ that outlive the call to $q$. They are live before the call and still live after the call.

The motivation is that, in the region-annotated program, the region variables of $p$ that are mapped to by those in $\operatorname{born} R(q)$ will get bound to a region during $q$ and are still bound after $q$, the ones mapped to by those in $\operatorname{deadR}(q)$ are bound before the call to $q$ and are safely removed during $q$, and the ones mapped to by those in outlived $R(q)$ are bound before the call and maintain their bindings throughout the call. 
We call the set of the region variables that are local to $p$ (not reachable from input or output variables), $\operatorname{local} R(p)$. The calling contexts of a procedure influence what a procedure can do to its non-local region variables. Therefore when analysing a procedure $p$, region variables that need to be live after the call to $q$ in $p$ are not allowed in $\operatorname{deadR}(q)$ but are put in outlivedR $(q)$ : the regions should not be reclaimed during the call. Similarly, regions already live before the call to $q$ should not be in $\operatorname{born} R(q)$ but in outlivedR $(q)$ : the regions already exist before the call. Also region alias has its impact. A typical case is when a procedure, e.g., $q\left(X_{1}, X_{2}\right)$, with $R_{X_{1}} \neq R_{X_{2}}$ is called as $q\left(Y_{1}, Y_{2}\right)$, with $R_{Y_{1}} \equiv R_{Y_{2}}$. Then $R_{X_{1}}$ and $R_{X_{2}}$ are neither in $\operatorname{dead} R(q)$ nor in $\operatorname{born} R(q)$.

Each procedure has exactly one born $R$ set and one deadR set that are suited for the most restrictive context. If the procedure is called in a less restrictive context, it will be the case that creation and removal will happen outside the call.

In our running example, produce is called in two calling contexts, so we will look at how its bornR and deadR sets are computed. Initially, born $R$ (produce $)=\{R 5\}$ because $\mathrm{R} 5$ is reached from the output argument $\mathrm{Y}$ and deadR(produce $)=\left\{R_{4}\right\}$ because R4 is reached from the input argument X. The first calling context is at (4) in produce, where $\alpha\left(R_{4}\right)=R_{4}$ and $\alpha(R 5)=$ $R 5$. It should be straightforward to see that $\mathrm{R} 4$, which is reached by $\mathrm{X}$ and $\mathrm{Xs}$, is live before the call and is no longer live after it. So it is safe that R4 ceases to be live in this call. For R5, which is reached from Y and Ys, it is not yet live before (4) and is live after. So R5 can become live in this call. The other calling context of produce is at (3) in q. Here we have $\alpha\left(R_{4}\right)=R 1$ and $\alpha(R 5)=R 2$. Again we can see that R1 is live before and not live after (3), meaning that it can become dead in the call. R2 is not live before and is live after (3) so it can become live in this call. Therefore finally, we have $\operatorname{born} R(\operatorname{produce})=\{R 5\}$ and deadR $($ produce $)=\{R 4\}$.

\section{Good Same-Edges}

The same-edges in our new region points-to graphs indicate regions that are candidates for reuse. For a procedure q, we keep a sameedge $s(R 1, i, R 2)$ with the intention that at the program point $i$ we should reuse the region bound to by $\mathrm{R} 1$ for R2, i.e., we make R2 bound to the region currently bound to by R1 and R1 is considered unbound after that. However, it is only safe to do that if R2 is not yet bound before $i$, if $\mathrm{R} 1$ is not live after $i$, and if $\mathrm{q}$ is allowed to manipulate them. This implies the following safeness conditions for a same-edge $s(R 1, i, R 2)$.

DEFINITION 7.1. [Safeness conditions] A same-edge $s(R 1, i, R 2)$ in the region points-to graph of a procedure $q$ is good if the following conditions are satisfied.

1. $R 1 \in L R_{\text {before }}(i) \backslash L R_{\text {after }}(i)$,

2. $R 2 \in L R_{\text {after }}(i) \backslash L R_{\text {before }}(i)$,

3. $R 1 \in \operatorname{deadR}(q) \cup \operatorname{local} R(q)$,

4. $R 2 \in \operatorname{bornR}(q) \cup \operatorname{localR}(q)$.

The first two conditions can be seen as the local liveness requirements, while the global liveness requirements are in the last two. We call the same-edges that satisfy the above conditions the good ones. Otherwise there is no point keeping them and their regions can be unified after all.

In Figure 8 we extend the running example with two calling contexts of q to show the effect of good and bad same-edges.

In the first calling context, $\mathrm{X}$ is no longer used after the call to q. So we have $\operatorname{dead} R(q)=\{R 1\}$, $\operatorname{born} R(q)=\{R 3\}$, and $\operatorname{local} R(q)=\{R 2\}$ (the reader may want to consult Figure 5 for the region variables). For produce, deadR(produce $)=\left\{R_{4}\right\}$ and $\operatorname{born} R(q)=\{R 5\}$. This program can be annotated as in Figure 9 .

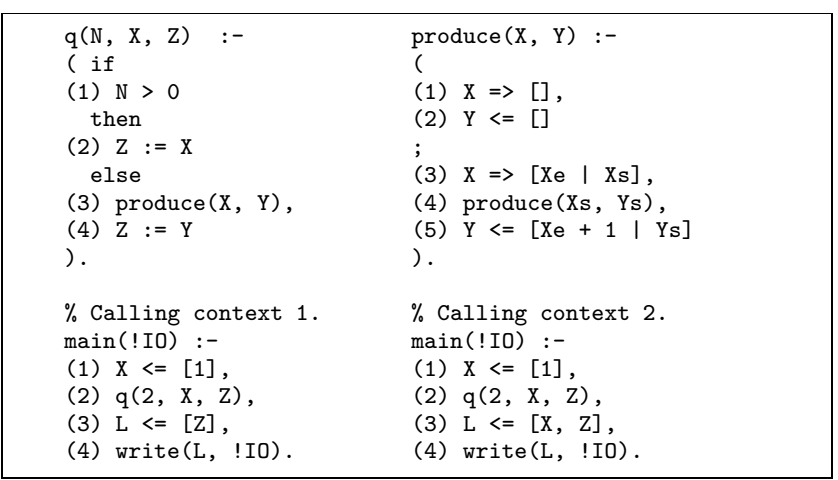

Figure 8: The running example extended with calling contexts of $q$.

Its behaviour is that the region of $\mathrm{X}$, namely R6, is removed in the

\begin{tabular}{|c|c|c|}
\hline $\begin{array}{l}\mathrm{q}(\mathrm{N}, \mathrm{X} @ \mathrm{R} 1, \mathrm{Z} @ \mathrm{R} 3) \\
\text { ( if } \\
\text { (1) } \mathrm{N}>0 \\
\text { then } \\
\text { (2) Z@R3 :== X@R1, } \\
\text { else } \\
\text { (3) produce (X@R1, } \\
\text { (4) Z@R3 :== Y@R2, } \\
\text { ). }\end{array}$ & $\begin{array}{l}\text { main } \\
\text { (1) } \mathrm{X} \\
\text { (2) } \\
\text { (3) } \\
\text { (4) }\end{array}$ & 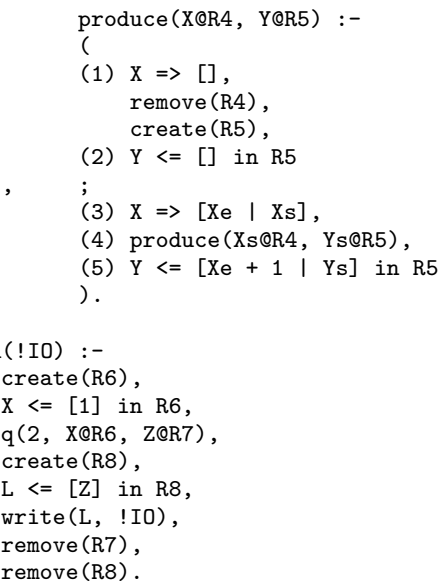 \\
\hline
\end{tabular}

Figure 9: Reuse region-annotated version for calling context 1.

call to q. This is safe because $X$ is no longer live after the call. $R 7$ is bound either to the region $\mathrm{R} 6$ or to a new region created by the call to produce in q depending on which execution path is taken in q. But this does not matter because from outside of $q$ we can assume that $\mathrm{R} 6$ is dead and R7 becomes live. And we only need to reclaim R7 afterwards.

In the second calling context, because $\mathrm{X}$ is still live after the call to $\mathrm{q}$, the call is no longer allowed to remove R6. This means that $\mathrm{R} 1$ is eliminated from deadR $(q)$ (i.e., R1 is now in outlived $R(q)$ ) and therefore $\mathrm{R} 4$ is excluded from deadR(produce). The annotated version now is in Figure 10. In produce there is no remove(R4) after (1) and in main remove (R6) is added after (4). If the program follows the first execution path in q, R6 and R7 are bound to the same region. Therefore that region will be wrongly removed twice in main. In general, we cannot guarantee which execution path is taken at runtime. Therefore in this case it is not safe to make use of the (bad) same-edges.

A safe way to handle this situation is to force $\mathrm{X}, \mathrm{Y}$, and $\mathrm{Z}$ in $\mathrm{q}$ into the same region as in the eager approach in [4]. The annotated program is in Figure 11. If we used the eager version for the first calling context, we would miss the chance to reuse the memory of $\mathrm{X}$ whenever the second execution path of $\mathrm{q}$ is taken.

So after the region liveness analysis we will re-examine the region points-to graphs to eliminate the bad same-edges based on 


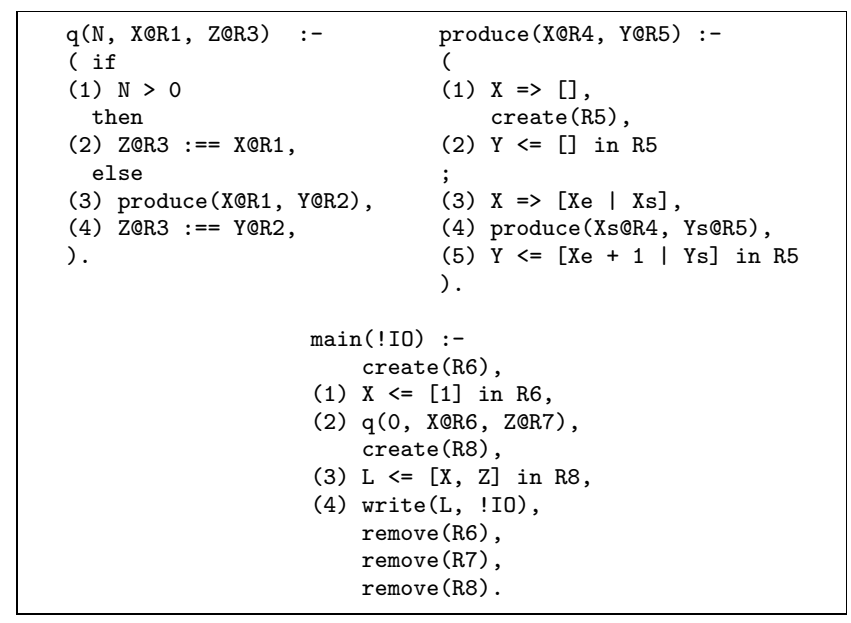

Figure 10: Wrong reuse region-annotated version for calling context 2 .

\begin{tabular}{|c|c|c|}
\hline $\begin{array}{l}\mathrm{q}(\mathrm{N}, \mathrm{X} @ \mathrm{R} 1, \mathrm{Z} @ \mathrm{R} 1) \\
(\text { if } \\
\text { (1) } \mathrm{N}>0 \\
\text { then } \\
\text { (2) } \mathrm{Z}:=\mathrm{X}, \\
\text { else } \\
\text { (3) produce(X@R1, } \\
\text { (4) } \mathrm{Z}:=\mathrm{Y} \\
\text { ). }\end{array}$ & $\begin{array}{l}:- \\
\\
\text { Y@R1), } \\
\\
\\
\text { main (! I0) } \\
\text { create } \\
\text { (1) } \mathrm{X}<=[ \\
\text { (2) } \mathrm{q}(0, \mathrm{X} \\
\text { create } \\
\text { (3) L }<=[ \\
\text { (4) write ( } \\
\text { remove } \\
\text { remove }\end{array}$ & 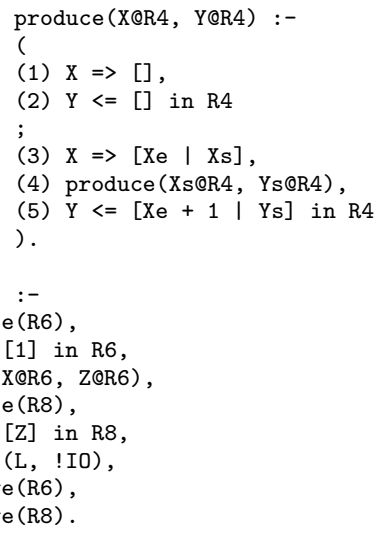 \\
\hline
\end{tabular}

Figure 11: Region-annotated version with the eager approach.

the above safeness conditions. If some same-edges are excluded from the region points-to graph of a procedure $q$, it means that the region points-to graph of $q$ changes. The change will affect the region points-to graphs of the callers of $q$ and the $\alpha$ mappings at the sites where $q$ is called. Therefore we need to re-run the interprocedural region points-to analysis for the callers of $q$, i.e., all procedures in the same SCC as $q$ and the ones in the SCCs that depend on $q$ 's SCC. We again can reach the fixpoint for each SCC in bottom-up order. The live variable analysis does not need to be run again. However, the derivation of live region variables at the program points in $q$ is based on its region points-to graph and will need to be done again. The change of the local region liveness in $q$ likely causes changes to the global region liveness of the procedures it calls, i.e., all procedures in the same SCC as $q$ and the ones in the SCCs on which the SCC of $q$ depends. So we also need to recompute the born $R$ and deadR sets of them.

We repeat the above process until no more same-edge is removed. At that time, all the remaining same-edges imply that reuses can safely happen. The whole algorithm is specified in Algorithm 5 in which safe (se) checks whether the same-edge se satisfies the safeness conditions or not. $\operatorname{dep}(q)$ computes the strongly con- nected component containing $q$ and also the components that depend on the SCC of $q$. invert_dep $(q)$ computes the strongly connected component containing $q$ and also the components on which the SCC of $q$ depends. local_region_liveness $(q)$ computes the sets of live region variables before and after program points in $q$ (Section 6.1). global_region_liveness $(q)$ applies the analysis in Section 6.2 to $q$ to re-calculate the bornR, deadR, and outlivedR of the procedures $q$ invokes.

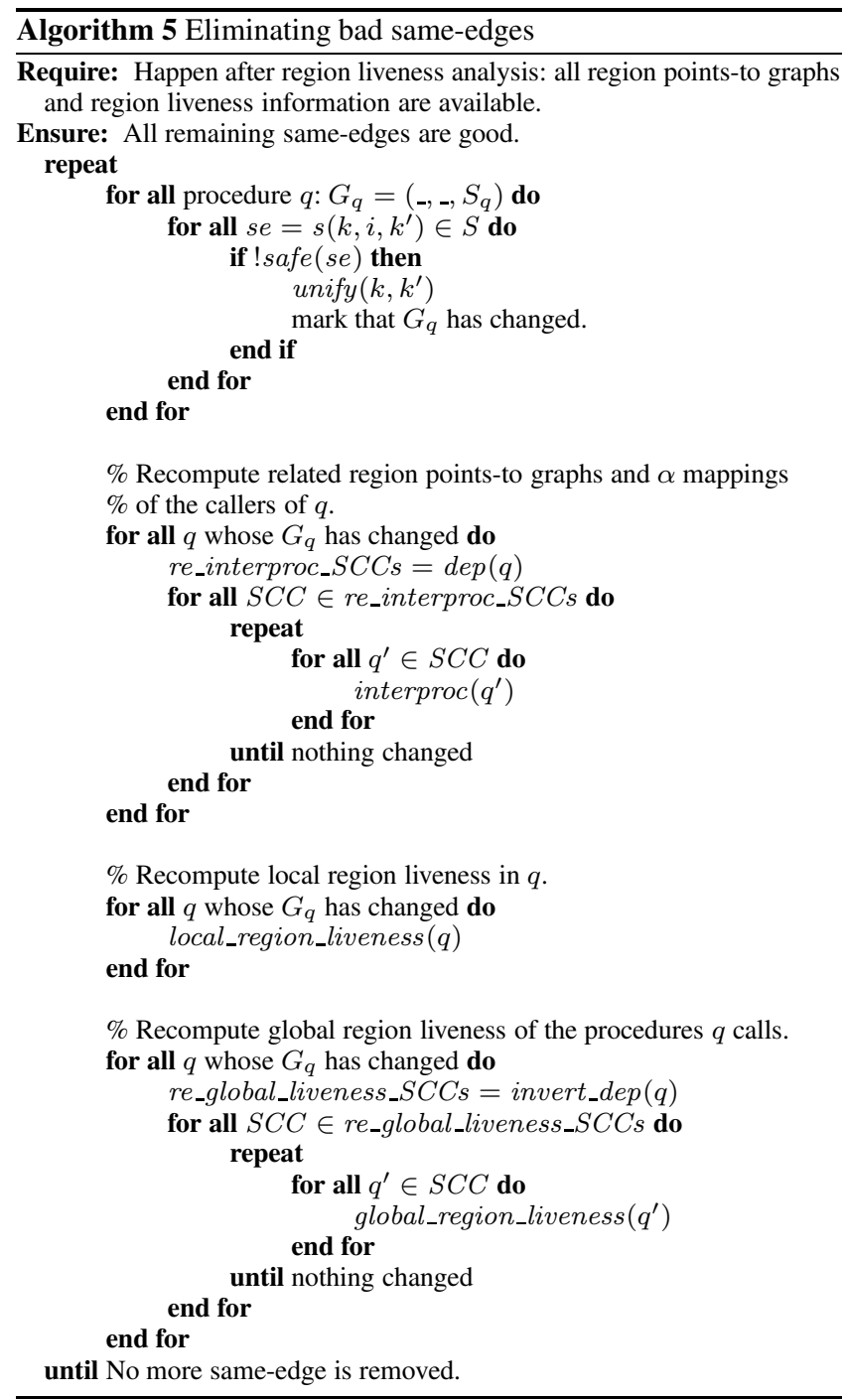

\section{Transformation}

A region-annotated program is finally generated by a program transformation of the original program taking into account the information derived by the region points-to analysis and the liveness analysis.

The transformations for adding extra arguments for region arguments, for annotating construction unifications with region variables, and for introducing the create and remove instructions are exactly the same as presented in [5].

A procedure can safely manipulate the region variables in its bornR, deadR, or localR. Ones in localR are local to the procedure. For the non-local ones in born $R$ and deadR we are sure that the global context of the procedure does not interfere with them, as 


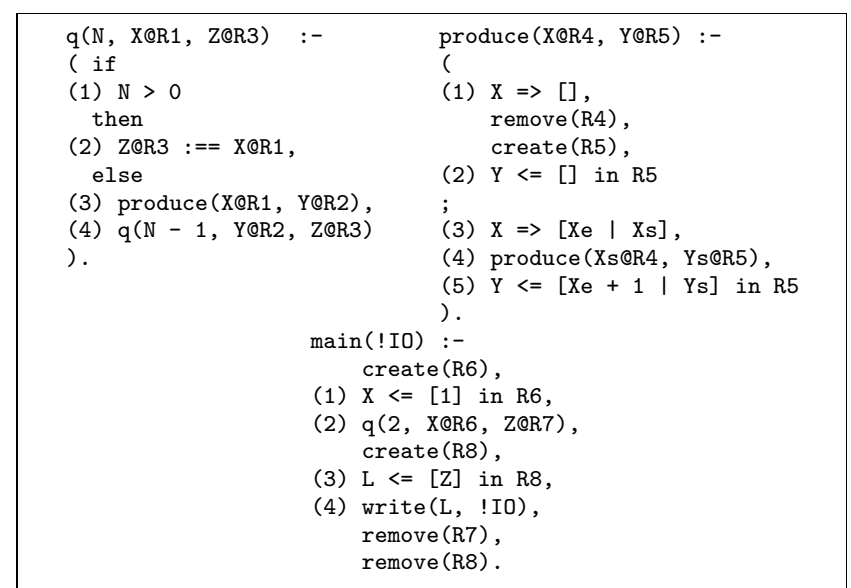

Figure 12: No explicit "reuse" after procedure calls.

the liveness and region aliasing have been taken into account when computing bornR and deadR. Consider a region variable $\mathrm{R}$ that the procedure can manipulate. In general a create $(R)$ is inserted before the program point where $\mathrm{R}$ becomes live, i.e., it is not live before but live after the point and the literal at the point does not create it. For removal, there are two cases. If $\mathrm{R}$ becomes dead because it is live before but not live after the point, we add remove (R) after the point if the literal at the point does not remove $R$. If $R$ becomes dead since it is live after the point but is not live before the next point in an execution path, we add remove $(R)$ before the next point.

For our new analysis we need to introduce the region-annotated assignment. Because all the remaining same-edges are good ones, if the literal at the program point $i$ of the same-edge $s(R 1, i, R 2)$ is an assignment $\mathrm{X}:=\mathrm{Y}$ where $\mathrm{X}$ is in $\mathrm{R} 2$ and $\mathrm{Y}$ is in $\mathrm{R} 1$, we just replace it with $\mathrm{X} @ \mathrm{R} 2:==\mathrm{Y} @ \mathrm{R} 1$ at $i$. If the literal is a procedure call it means that the reuse happens inside the call and we do not need to make any change.

We can see the effect of same-edges at procedure calls when changing the code of $q$ by replacing the assignment at (4) with a recursive call as in Figure 12. Nothing other than region arguments is added at (4).

\section{Discussion and Concluding Remarks}

In the paper we have presented an improvement for the region analysis and transformation in [4] that results in better memory reuse for a certain class of programs. By making the region pointsto analysis path-sensitive (in the sense that it distinguishes different local execution paths in a procedure) we achieve a more precise region model of memory use. This information then is verified against the liveness information to make sure that the resulting transformation is sound. We formulate the local and global liveness safeness conditions for this purpose.

Implementing the improvement presented in this paper is part of future work. We believe that the runtime support for regions in [6] is able to support region reuse with little change. Other than implementing the regions themselves and the region instructions, the runtime support for regions has two tasks in supporting backtracking. First, it provides protection for regions that are removed during forward execution if they are still needed when backtracking happens, ensuring that these regions will only be reclaimed when they are not used in any future computation. Second, it allows instant reclaiming of new regions and new allocations into old regions at choice points. The essential elements in the support are the timestamping of the regions when they are created in order to distinguish old and new regions and the estimation of the sets of regions that are created, removed and allocated into by a goal. The region reuse affects none of these because the time-stamp of a reused region is intact when the reuse happens and because of the fact that the region is reused the created, removed, and allocated region sets of any goals are not changed.

The class of programs that potentially benefit from this novel extension includes state transition programs in which a sequence of states are computed iteratively and typically we are only interested in the last state. In the set of benchmark programs used in [6], there are two typical examples of this for which the region reuse improvement should be beneficial, namely life and dna. We discuss these two programs in detail but before that we will explain how the experiments have been done.

As we do not have an implementation of region reuse yet, we mimic the memory behaviour of the two programs as if region reuse had been used by changing their source. Recall that in Section 2 we introduced region reuse to avoid copying terms at the moment of an assignment unification. Therefore we modify the benchmarks by adding an explicit copying at the place where our new approach would introduce a region reuse assignment. After that we can use the existing RBMM system in [6] to execute them. By doing so we can study the effects of region reuse on memory consumption. Note that for the discussion here, we manually introduce copying procedures that make the desired copies. The existing region analysis [6] takes into account the copying procedure and then can decide whether the original and the copied ones can be kept apart or not. For example, for a list we copy both the list skeleton and the elements, the region analysis decides for the program as a whole whether the old and the new skeletons as well as the old elements and the new elements are in the same regions or not. In summary, we can check whether region reuse can be done and whether it is beneficial for life and dna by adding the copying and by using the existing RBMM system. The profiling information about memory consumption of these programs allows us to measure the effects of region reuse.

The life benchmark encodes the program Game of Life in which a new generation is generated from a previous one based on a set of production rules. Its code follows the pattern of $q$ and produce in Figure 12 in which produce implements the production rules and $\mathrm{q}$ is the loop for computing next generations and in the base case of the loop there is an assignment between the input and output generations. A generation is represented by a list of live cells, each cell is represented by its row and column in a $20 \times 20$ board. To store a generation we need two regions, one for the skeleton and the other for the cells. In the program the list skeletons of two successive generations are independent while their cells may share. The program creates 50 generations from a starting one of 100 live cells. As reported in [6], without region reuse, the program needs maximally 8208 words to run. A closer look into the program reveals that the skeletons of all the generations are stored in one region with a size of 6486 words, which is the biggest region of the program. The related cells are stored in another region. Because they are actually shared among the generations, region reuse cannot help separate them into different regions. However, region reuse can help with splitting the skeletons and then reclaiming them. We mimic region reuse for life by replacing the assignment between two generations (like the one at (2) in q) with a call to a procedure that copies the list skeleton. By evaluating the region reuse-mimicking program we measure that the maximal number of words used is reduced by $77 \%$ to 1856 words because the garbage consisting of the skeletons of the old generations and of temporary data created during the process of generating a new one is timely reclaimed. 


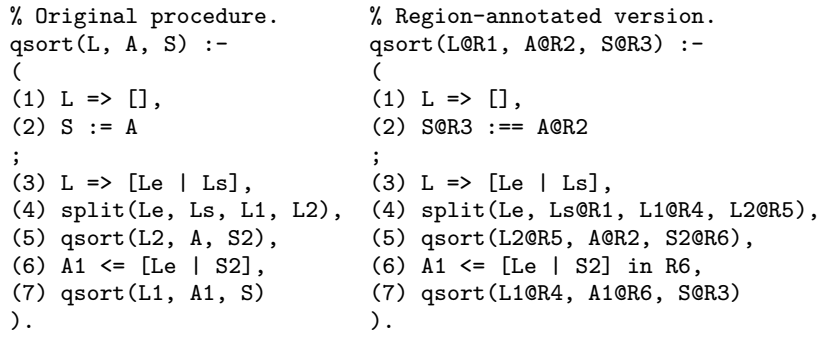

Figure 13: A chance for optimization.

The dna computes the similarities of a given DNA sequence against each sequence from a test set of 1000 DNA sequences. The similarity between two sequences is derived from the similarity of each element in one sequence against each element in the other sequence. All the ever-computed similarities are put into one region that is actually the biggest region in the program consisting of more than $4 \mathrm{M}$ words. The maximal number of words in use during a run of the program is about $4.6 \mathrm{M}$.

The procedure to compute the similarity between two sequences again has a pattern similar to the code in Figure 12. The base case has an assignment between the input and output similarities. The question is whether region reuse could be done here. Our region analysis of the changed program in which the region-annotated assignment is mimicked by a call to a procedure that copies the input similarity to the output one decides that the similarities still cannot be put in two different regions. Looking in detail at the program we see that the reason is because the output similarity is also accumulated into a temporary list for future computations. All the elements of the list have to be in one region (due to our treatment of recursive types) and this forces the potential reuse candidates to be in one region too. With that in mind, to make use of region reuse, we have to modify the mimicking program by adding more copying, i.e., a copy of the output similarity is added to the list. We call the resulting program dnacrow. After this nontrivial modification, the region analysis of dnacrow can keep the similarities in different regions. Running the program shows that the maximal number of words used goes down $89 \%$ to less than $0.5 \mathrm{M}$ words, and that the size of the biggest region is now of less than $0.43 \mathrm{M}$ words. The biggest region is the region of the skeleton of the tree data structure used to store the resulting similarities.

In [3] a so-called region-friendly version of dna, rdna, has been proposed. In rdna, a similarity between two DNA sequences is copied before it is put in the tree. The maximal number of words used in rdna, more than $0.5 \mathrm{M}$, is a little bigger than that in dnacrow. However, as less copying happens in rdna, we can expect less runtime overhead due to copying. This shows that copying can be done at several places with different impact on memory consumption and runtime performance.

The attentive reader might get worried about procedures such as qsort in Figure 13. In the approach in [4] all the variables involved with the accumulating parameter and the result are put in the same region. This is perfect because the accumulator is gradually built up to become the final result. Our new approach seems to be spoiling this. First notice that A1 and S2 are in the same region $R 6$ due to the construction. In the first execution path we have a same-edge at (2) from the region R2 of A to the region R3 of S. In the second execution path we have a same-edge at (5) from R2 to R6 and a same-edge at (7) from R6 to R3. All sameedges are "safe". The resulting region-annotated program has a region-annotated assignment at (2). When we mimic the region- annotated assignment by a copying of the list skeleton, we see that region reuse can be done and that the maximal number of words used is the same as with the existing RBMM system, thus optimal. However, this instruction is sheer overhead. It would be removed by the following optimization step. For qsort in all execution paths we have a same-edge path from the region R2 of A to the region R3 of S. Thus, we could put all the involved variables again in one region. Note that the optimization condition does not hold for e.g., $\mathrm{q}$ in Figure 12. More programs will also have to be studied in order to know the relevance and the impact of this optimization step.

Birkedal et al. [1] present Storage Mode Analysis that targets the same class of programs. Being an extra phase after the region inference in [8] that puts all such states into the same region, the analysis then aims to reset the region before each iteration if it is safe to do so. The decision is also based on liveness information. However for Game of Life, it requires manually rewriting the program with a copying function so that the resetting is possible [2]. Henglein et al. [2] develop an expressive region type system that can accept several region-annotated versions for a program. Their region inference based on that type system also can produce an annotated version with the same region behaviour for Game of Life as ours, without requiring rewriting. One interesting open problem with their region type system is to have a strong region inference that, among a number of accepted annotated programs, can choose to generate an optimal one. We start from an analysis algorithm that performs well generally and extend it to obtain better results in a popular pattern of code, which is well known to be difficult for RBMM. By mimicking region reuse using copying procedures, we showed that it can effectively reduce memory consumption however there exist cases where the programs need to be made more region-friendly before it can be done. Whether this change can also be automated is an open problem.

\section{Acknowledgments}

We would like to thank the referees for the constructive comments. Quan Phan is supported by FWO project G.0221.07.

\section{References}

[1] L. Birkedal, M. Tofte, and M. Vejlstrup. From region inference to von Neumann machines via region representation inference. In Principles of Programming Languages., pages 171-183. ACM Press., 1996.

[2] F. Henglein, H Makholm, and H. Niss. A direct approach to controlflow sensitive region-based memory management. In Principles and Practice of Declarative Programming., pages 175-186. ACM Press., 2001.

[3] H. Makholm and K. Sagonas. On enabling the WAM with region support. In Proceedings of the 18th International Conference on Logic Programming. Springer Verlag., 2002.

[4] Q. Phan and G. Janssens. Static region analysis for Mercury. In Proceedings of the 23rd International Conference on Logic Programming, pages 317-332. Springer, 2007.

[5] Q. Phan and G. Janssens. Region-based memory management for Mercury programs. Part 1: Region analysis and transformation. Technical Report CW540, Department of Computer Science, Katholieke Universiteit Leuven, 2009.

[6] Q. Phan, Z. Somogyi, and G. Janssens. Runtime support for regionbased memory management in Mercury. In Proceedings of the 2008 International Symposium on Memory Management, pages 6170. ACM Press., 2008.

[7] Z. Somogyi, F. Henderson, and T. Conway. The execution algorithm of Mercury, an efficient purely declarative logic programming language. The Journal of Logic Programming, 29(1-3):17-64, OctoberDecember 1996.

[8] M. Tofte and J.-P. Talpin. Region-based memory management. Information and Computation., 132(2):109-176, February 1997. 